\title{
CONSTRAINT ASPECTS OF THE EVALUATION OF FATIGUE TEST RESULTS IN PARIS REGION
}

The growth of the fatigue crack is dependent on the crack growth resistance of the material as a bulk property. The fatigue crack growth properties of a material are usually described by the correlation between a crack propagation rate, and a corresponding range of the stress intensity factor (Paris-Erdogan law). Formulated modified Paris-Erdogan law estimates quantitatively the changes of the fatigue crack propagation rate due to different level of constraint. In-plain constraint was described by T-stress value. The validity of the approach suggested has been proved by the comparison of measured and calculated values of the crack propagation rate and it was found that experimental values correspond well with those numerically predicted. The results presented makes it possible to relate experimentally measured data obtained from specimens with different geometries and thus contribute to more reliable estimates of residual fatigue life of structures.

\section{Introduction}

The fatigue life of structures can be divided into two parts. The first one corresponds to a crack initiation period and the second one corresponds to a crack growth period until a complete failure. Crack initiation and growth are different phenomena and their influence on the total service life of structures depends on many circumstances.

The fatigue life of an unnotched specimen in high cycle fatigue regime is largely covered by the crack initiation period. The crack growth period could almost be disregarded and often neglected. Nevertheless there are situations where the fatigue crack propagation period plays a decisive role and has to be carefully analysed. Let us mention two such examples. The crack initiation period might be shorter due to the existence of stress concentrators (such as existing cracks, notches, material discontinuities...). In this case the fatigue crack propagation in components can start practically even without a crack initiation period. Another category of problems connected directly with fatigue crack propagation is related to a fatigue crack growth monitored by periodic inspections. The purpose of the inspections is to discover fatigue cracks before they become dangerous and to do this it is necessary to know how fast a crack is growing in order to set timely inspection periods. In this situation is the knowledge of the fatigue crack propagation rate of paramount importance.

The growth of the fatigue crack is then dependent on the crack growth resistance of the material as a bulk property. The fatigue crack growth properties of a material are usually described by the correlation between a crack propagation rate, $d a / d N$, and a corresponding range of the stress intensity factor $\Delta K$. The correlation is usually expressed by an empirical function based on test data. The most popular relation between $d a / d N$ and $\Delta K$ can be described by a power function (Paris-Erdogan law), see e.g. [1], [3].

$$
d a / d N=C \Delta K^{m}
$$

with $C$ and the exponent $m$ as material constants. The fact that $C$ and $m$ are material parameters means that for a given material and set of test conditions, the growth rate should depend only on $\Delta K$, i.e. the eq. (1) represents a material curve. Eq. (1) does not account for $R$-effect on crack growth, neither for the asymptotic behavior near the threshold and the final failure regions. In order to apply eq. (1) to predict the lifetime of structures, the corresponding material data, $C$ and $m$, must be known. The methods for conducting fatigue crack growth testing and evaluation of parameters $C$ and $m$ are specified in standards (e.g. ASTM E 647 [2]). The testing can be conducted on any type of a test specimen or structural component. Usually a compact tension specimen (CT), center cracked tension specimen (CCT) or three point bend specimen (3PB) are used for generating material $d a / d N$ data. Some results of the fatigue crack propagation tests using several different specimens showed significant dependence on specimen geometry, e.g. [4], [8], [9]. Thus the $C, m$ values cannot be considered as material constants, in all cases and their values can depend on geometry of the specimen used for their determination. This fact makes questionable the transferability of the laboratory data to real structural components. The aim of the contribution is to suggest an approach how to quantify the effect of specimen geometry on the fatigue crack propagation rate.

\section{Experimental observations}

The influence of the specimen geometry on the fatigue crack propagation rate (FCPR) taken from paper [9] is presented on Fig.1.

As a result, the fatigue crack propagation rate was found to be greater for $\mathrm{CCT}$ specimen than for the $\mathrm{CT}$ one. In our laboratory

\footnotetext{
* Zdeněk Knésl, Pavel Hutař, Stanislav Seitl

Institute of Physics of Materials, Žižkova 22, 61662 Brno, E-mail: knesl@ipm.cz
} 


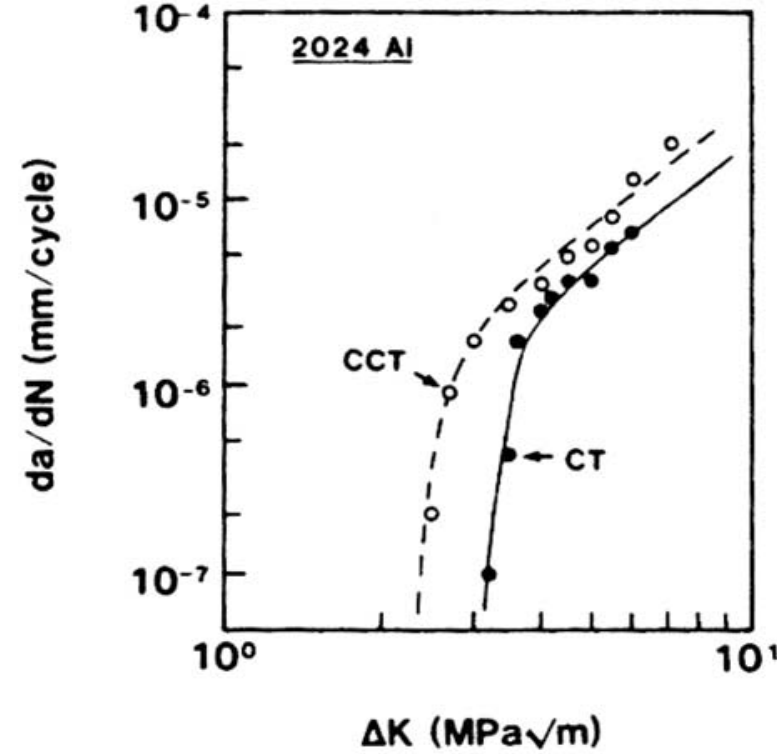

Fig.1 Effect of specimen geometry on crack behavior near threshold of 2024 aluminum alloy at $R=0.1$. Taken from reference [9].

0.45\% carbon steel was used for similar experiments, see Fig. 2 . The measurements are in agreement with previous authors. All experiments were performed at the same conditions - room temperature and loading stress ratio $R \sim 0.1$ (i.e. $\Delta K_{I} \sim K_{I}$ ). The crack length was measured optically with a resolution of $0.01 \mathrm{~mm}$.

Discrepancies in the fatigue crack growth rate in the Paris region can be explained by constraint based fracture mechanics. In conventional one parameter fracture mechanics the stress field around the crack tip is described by one parameter. In two parameter constraint based fracture mechanic the stress field is expressed by means of the two parameters, the stress intensity factor $K_{I}$ and the $T$-stress. The $T$-stress quantifies the effect of the crack tip constraint, which depends on the outer geometry of structure. Both these parameters are related to the first and second term of Williams expansion [10]. The stress field near the crack tip for a normal mode of loading can then be expressed as

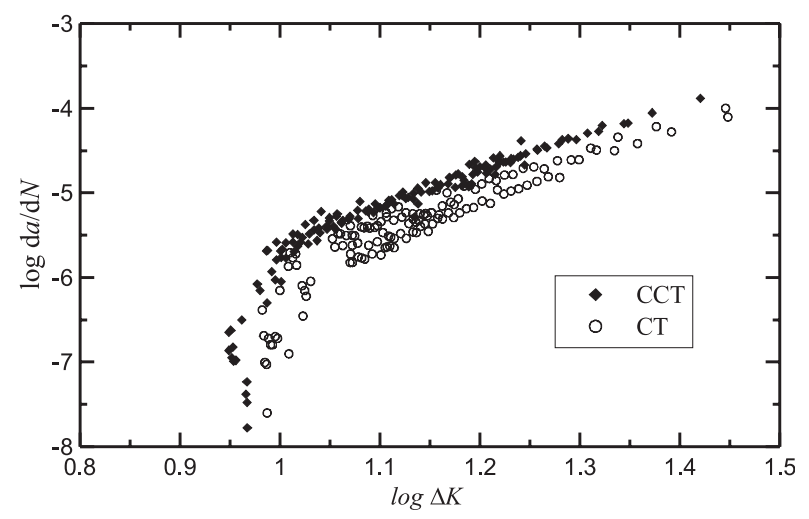

Fig. 2 Influence of the constraint on the threshold values and fatigue crack propagation rate of $0.45 \%$ carbon steel at $R=0.1$ [4].

$$
\sigma_{i j}=\frac{K_{I}}{\sqrt{2 \pi r}} f_{i j}(\theta)+T \delta_{1 i} \delta_{1 j},
$$

where $K_{I}$ is the stress intensity factor, $T$ is $T$-stress and $f_{i j}$ is a known function of the polar angle $\theta$.

The center cracked plate tension specimen (CCT) represents geometry with negative values of the $T$-stress (loss of constraint), which is practically independent of the crack length [7]. On the other hand the compact tension (CT) specimen corresponds to geometry where the $T$-stress values are positive and depend more significantly on the crack length [7]. Therefore, the fatigue crack propagation rate was found to be higher for a structure with a negative value of $T$-stress (CCT specimen) than for a structure with a positive value of T-stress (CT specimen).

\section{Constraint based description of fatigue crack behavior}

During cyclic loading the monotonic and cyclic (or reversed) plastic zone is created ahead of the growing crack. The size and the properties of the plastic zone are decisive for the propagation of a fatigue crack and control its behaviour [3].

The results of finite element computations in Fig.3 show that for the same value of $K_{I}$ (we assumed the normal mode of loading only), the crack tip plastic zone size and shape are different for different values of the $T$-stress. Typically, a negative value of the $T$ stress, $T<0$, causes enlargement of the plastic zone as compared to the case where $T=0$. Moreover, for plane strain conditions, the plastic zone is rotated to the region ahead of the crack tip for negative values of $T$. The size of the plastic zone decreases for positive values of the $T$-stress, but the dependence is not so pronounced. Note that $T=0$ corresponds to the assumptions of one parameter fracture mechanics.
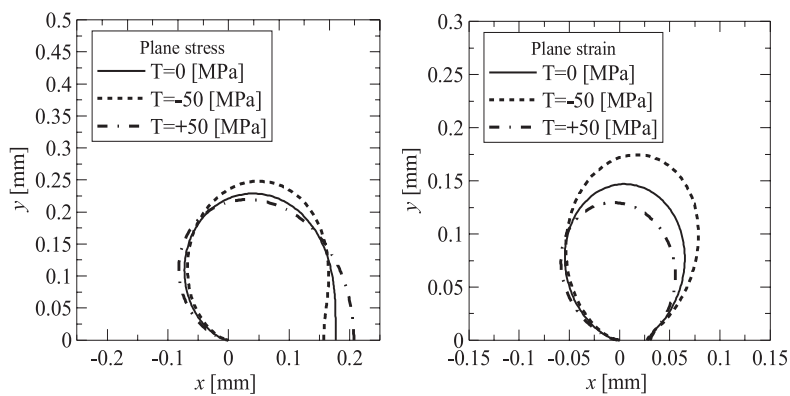

Fig.3 Dependence of the size and shape of the crack tip plastic zone on the T-stress value (a) plane stress conditions (b) plane strain conditions.

Accepting the assumption that the size of the plastic zone is dominant for the propagation of a fatigue crack, it is possible to estimate quantitatively the changes of the fatigue crack propagation rate due to different constraint level [4]. 
Under small scale yielding (SSY) conditions there is a single valued relation between the size of the plastic zone at the crack tip and the value of the corresponding stress intensity factor controlling the fatigue crack propagation rate. Following this, one of the parameter, say $S_{P}=S_{P}\left(K_{I}\right)$, which characterizes the area of the plastic zone, can be used as the controlling variable. Paris law can be then rewritten as a function of $S_{P}$ in the form:

$$
d a / d N=C\left(\sigma_{0} \sqrt[4]{\frac{S_{P}}{\Psi(v)}}\right)^{m},
$$

where $\Psi(v)$ is a known function of the Poisson ratio $v$. Under the framework of one parameter linear-elastic fracture mechanics, equations (1) and (3) are equivalent. The assumption of a unique relationship between $d a / d N$ vs. $K_{I}$ independent of the geometry and the fact that the area of the plastic zone depends on the geometry of the specimen are contradictory. If the constraint expressed by the $T$-stress influences the size of the plastic zone, it may also influence the fatigue crack propagation rate $d a / d N$, and the $d a / d N$ vs. $\Delta K$ curve may depend on the geometry of the specimen. Thus eq. (3) considers the effect of the stress field at the tip of the crack, including the non-singular T-term, on the fatigue crack propagation rate.

The influence of the $T$-stress on the size of the plastic zone $S_{p}(K, T)$ was assessed using a modified boundary layer analysis, see, e.g., [5]. As a result of the numerical simulations, it can be seen that the relation between $S_{p}(K, T)$ and $S_{p}^{0}(K, T=0)$ depends on the hardening coefficient $n$ only very slightly and can be expressed in the form:

$$
S_{p}(K, T)=\lambda\left(T / \sigma_{0}\right)^{4} S_{p}(K, T=0),
$$

where

$$
\lambda\left(T / \sigma_{0}\right)=1-0.85\left(\frac{T}{\sigma_{0}}\right)^{3}+0.52\left(\frac{T}{\sigma_{0}}\right)^{2}-0.3\left(\frac{T}{\sigma_{0}}\right),
$$

Using the Paris-Erdogan equation (1) and (5) we get the following form:

$$
d a / d N=C\left[\lambda\left(T / \sigma_{0}\right)\right]^{m} K^{m}
$$

Eq. (6) represents a modified form of the Paris-Erdogan equation and makes it possible to account the constraint effect on the fatigue crack propagation rate. $C$ and $m$ are material constants obtained for conditions corresponding to $T=0$. The value of the T-stress in (5) represents the level of constraint corresponding to the given specimen geometry. Eq. (6) can be expressed in the form corresponding to standard Paris - Erdogan equation.

$$
d a / d N=C^{*}(K)^{m},
$$

where $C^{*}=C_{0}\left[\lambda\left(T / \sigma_{0}\right)\right]^{m}$. It follows from eq.(7) that $d a / d N$ vs. $K_{I}$ curves corresponding to different specimens geometry with different values of $T$-stress create a set of parallel straight lines on a $\log -\log$ scale. This corresponds to experimental results presented in the paper. The "constraint based" material parameter $C^{*}$ can be expressed in form:

$$
C^{*}=C^{0}\left(1-0.85\left(\frac{T}{\sigma_{0}}\right)^{3}+0.52\left(\frac{T}{\sigma_{0}}\right)^{2}-0.3\left(\frac{T}{\sigma_{0}}\right)\right)^{\mathrm{m}},
$$

where $C^{0}$ is the experimentally obtained material constant for $(T=0), T$ is a $T$-stress and $\sigma_{0}$ is a yield stress of controlled structure.

\section{Conclusions}

The influence of in-plane constraint on the propagation rate of the fatigue crack in the Paris region has been investigated. After assumption that the size of the plastic zone is dominant for the propagation of the fatigue crack modified Paris-Erdogan equation has been introduced. The results of the paper can be summarized in the following way:

The effect of the specimen geometry on the relationship between the propagation rate of a fatigue crack and the range of the stress intensity factor amplitude might make questionable the uniqueness of the $d a / d N$ vs. $\Delta K$ curve for different geometries, which is essential if laboratory data are to be transferred to engineering applications.

Formulated modified Paris-Erdogan law estimates quantitatively the changes of the fatigue crack propagation rate due to different level of constraint. In-plain constraint was described by $T$-stress value. The validity of the approach suggested has been proved by the comparison of measured and calculated values of the crack propagation rate and it was found that experimental values correspond well with those numerically predicted.

Under small scale yielding conditions, the specimens with negative values of the $T$-stress exhibit a higher fatigue crack propagation rate than the specimens with positive values of $T$-stress at the same value of stress intensity factor range $\Delta K$. It was found that, depending on the material properties, geometry and loading of the specimen difference in $d a / d N$ due to constraint can reach $100 \%$ for extreme values of $T$, see eqs. $(5,6)$.

To overcome problems connected with influence of the specimen geometry on the fatigue crack propagation rate and to increase the reliability of the transferability of basic fatigue crack propagation data from laboratory specimen to engineering structures, the level of constraint of the specimen and the structure should be the same. If the constraint levels are different it is necessary to use eq. (8) and recalculate the value of $C$ parameter in Paris law.

\section{Acknowledgements}

This investigation was supported by grants 1QS200410502, 101/04/P001 and 106/06/P239. 


\section{References}

[1] ANDERSON, T. L.: Fracture mechanics, CRC Press, Boca Raton, Florida, 1995

[2] Annual Book of ASTM Standards 1999, Standard Test Method for Measurement of Fatigue Crack Growth Rates, E647-99, 1999

[3] DUGGAN, T. V.: A theory for fatigue crack propagation, Engineering Fracture Mechanics, Vol. 9, 1977, pp. 735-747

[4] HUTǍr, P., SEITL, S., KNÉSL, Z.: Quantification of the effect of specimen geometry on the fatigue crack growth response by twoparameter fracture mechanics, Material Science \& Engineering A, 387-389, 491-494, 2004

[5] HUTǍ̆, P., SEITL, S., KNÉSL, Z.: Effect of constraint on fatigue crack propagation near threshold in medium carbon steel, Computational Materials Science, Volume 37, Issues 1-2, 51-57, 2006

[6] KNÉSL, Z., SEITL, S., HUTAŘ, P.: Accounting for effects of constraint on propagation of fatigue crack, Damage \& Fracture mechanics VII, WIT press, 245-253, 2002

[7] KNÉSL, Z., BEDNÁŘ K., Two parameter fracture mechanics: calculation of parameters and their values (in Czech), Institute of Physics of Materials, Brno, 1998

[8] TONG J.: T-stress and its implications for crack growth, Engineering Fracture Mechanics, 69, 1325-1337, 2002

[9] VECCHIO, R. S., CROMPTON, J. S., HARTZBERG, R. W.: The influence of specimen geometry on near threshold fatigue crack growth, Fatigue Fract. Engng. Mater. Struct., 10, 333-342, 1987

[10] WILliamS, M. L.: On the Stress Distribution at the Base of Stationary Crack, Journal of Applied Mechanics, 24, 109-114, 1957 